\title{
Canakinumab in colchicine resistant familial Mediterranean fever and other pediatric rheumatic diseases
}

\author{
Mustafa Çakan`, Şerife Gül Karadağ ${ }^{\oplus}$, Nuray Aktay Ayaz ${ }^{\oplus}$ \\ Clinic of Pediatric Rheumatology, Kanuni Sultan Süleyman Research and Training Hospital, İstanbul, Turkey
}

\begin{abstract}
Background and objectives. The aim of this observational retrospective cohort study was to demonstrate indications and response rates of the patients with pediatric rheumatic diseases that used canakinumab.

Method. The files of the patients that used canakinumab between December 2012 and July 2017 were reviewed. Canakinumab was used in 29 patients. Diagnosis of the patients were; colchicine resistant familial Mediterranean fever (crFMF) (19 cases), hyperimmunoglobulin D syndrome-mevalonate kinase deficiency (HIDS-MKD) (3 cases), cryopyrin-associated periodic syndrome (3 cases), systemic juvenile idiopathic arthritis (sJIA) (2 cases), idiopathic recurrent pericarditis (1 case) and pyoderma gangrenosum (1 case).

Results. Canakinumab was used for $21.8 \pm 15.8$ months (6-54 months). crFMF patients had a female predominance; 16 girls and 3 boys. Mean age at the first symptoms of FMF was $2.8 \pm 2.2$ years. Mean number of attacks per year before colchicine was $18.7 \pm 6.9(10-36)$, after colchicine was $8.2 \pm 2.7$ (6-12) and after biologic agent the number dropped to $0.1 \pm 0.3(0-1)$. Canakinumab led to resolution of attacks in 3 HIDS-MKD cases. Two familial cold autoinflammatory syndrome patients were using canakinumab for 13 months with total remission. Chronic infantile neurological cutaneous articular syndrome patient did not show dramatic response to standard doses of IL-1 blockers and remission was achieved with high doses of canakinumab. Canakinumab led to the resolution of all systemic and articular manifestations in one sJIA case but the other sJIA case developed polyarticular joint involvement under canakinumab treatment. A severe pyoderma gangrenosum patient that failed dapson and anakinra, also failed canakinumab treatment that was used for 9 months. We have successfully treated a case of idiopathic recurrent pericarditis with canakinumab. Canakinumab was discontinued due to inefficacy only in two cases.
\end{abstract}

Conclusion. Overall efficacy of canakinumab was $93.1 \%$ in this study. No major adverse event was observed under canakinumab treatment. Canakinumab seems to be effective and safe in children with rheumatic diseases.

Key words: autoinflammatory diseases, canakinumab, colchicine resistant familial Mediterranean fever.

\begin{abstract}
Autoinflammatory diseases (AIDs) are a group of disorders characterized by recurrent attacks of fever, and systemic inflammation mainly mediated by the cells of the innate immune system. ${ }^{1}$ The world of autoinflammation is expanding rapidly with new diseases described every year since the first description of autoinflammation in 1999. ${ }^{2}$ Monogenic periodic fever syndromes
\end{abstract}

$凶$ Mustafa Çakan

mustafacakan@hotmail.com

Received 20th October 2018, revised 12th February 2019, 19th April 2019, accepted 3rd July 2019.
(MPFS), including familial Mediterranean fever (FMF), hyperimmunoglobulin $\mathrm{D}$ syndromemevalonate kinase deficiency (HIDS-MKD), cryopyrin-associated periodic syndrome (CAPS) and tumor necrosis factor receptorassociated periodic syndrome (TRAPS) comprise the most well defined group of AIDs and are characterized by attacks of fever, rash, polyserositis and systemic inflammation. FMF is the most common form of MPFS. ${ }^{1-3}$

The signs and symptoms in MPFS are due to overproduction of interleukin 1 (IL-1). Pyrin and NRLP3 mutations induce IL-1 $\beta$ release from myeloid cells by activating the inflammasome 
that regulates caspase- 1 activity. Description of the role of the inflammasome and IL- 1 in the pathogenesis of many AIDs have led to the use of more targeted treatment options. ${ }^{4,5}$ Colchicine is the mainstay therapy in FMF and most of the patients show dramatic response to it. ${ }^{6}$ However, nearly all of the patients with other MPFS do not respond to colchicine and IL-1 blockage is now regarded as the recommended treatment modality in these disorders. Also, around 5-10\% of FMF patients are nonresponsive to colchicine and there are many reports on beneficial effects of IL-1 blockers in colchicine resistant FMF (crFMF) patients. ${ }^{6-8}$ Canakinumab is a human monoclonal anti-IL-1 $\beta$ antibody that neutralizes the activity of IL-1 $\beta$ by binding to it. There are also two other IL-1 blockers; anakinra (a recombinant IL-1 receptor antagonist) and rilonacept (receptor fusion protein acting as IL-1 decoy receptor). ${ }^{9,10}$

The aim of this observational retrospective cohort study was to demonstrate diagnosis, demographic features, indications and response rates of the patients with pediatric rheumatic diseases that used canakinumab.

\section{Material and Methods}

This study was conducted in the pediatric rheumatology clinic of Kanuni Sultan Süleyman Research and Training Hospital. The files of the patients that canakinumab have been used between December 2012 and January 2017 were reviewed. Diagnosis, laboratory parameters, indications, duration of the treatment and response of the patients to the canakinumab were recorded. To be included into the study; the patient had to have either clinical and/or molecular diagnosis for that rheumatic disease and had to be using canakinumab for at least 6 months. All FMF patients fulfilled diagnostic criteria of Turkish pediatric FMF criteria. ${ }^{11}$ Also FMF, HIDS-MKD and CAPS patients fulfilled 'The Eurofever clinical diagnostic/ classification criteria for autoinflammatory periodic fevers' criteria set. ${ }^{12}$ In crFMF patients, FMF exacerbation was defined as having fever and abdominal and/or chest pain for at least 12 hours or more. Colchicine resistance was defined as having $\geq 3$ attacks for 6 consecutive months despite maximal colchicine dose. Initial colchicine dose was arranged according to age and started as $0.5 \mathrm{mg} /$ day for children $<5$ years of age, $1 \mathrm{mg} /$ day in two divided doses for children between 5 and 10 years of age and $1.5 \mathrm{mg} /$ day in three divided doses for children $>10$ years of age. Colchicine dose was increased $0.25 \mathrm{mg}$ every 3-6 months in partial or nonresponders as $2 \mathrm{mg} /$ day being the maximum daily dose. Canakinumab was started at a dose of $2 \mathrm{mg} / \mathrm{kg}$ in all indications and, if needed, the dose was gradually increased $(2 \mathrm{mg} / \mathrm{kg}$ per each increment) every 1 or 2 months until all signs and symptoms of that disease are controlled. Colchicine treatment was continued in all crFMF patients. The study was performed according to the tenets of Declaration of Helsinki and was approved by the local ethics committee (ethics approval number: KAEK/2018.3.27). Informed consent was taken from the legal guardians of the children.

Clinical and demographic characteristics were summarized by mean and standard deviation for continuous variables and count and percent for categorical variables. Statistical analyses were performed using the SPSS software package for Windows (version 22.0; SPSS, Chicago, IL, USA).

\section{Results}

Canakinumab was used in 29 patients during 4.5 years. The cohort consisted of 19 crFMF, 3 HIDS-MKD, 3 CAPS [2 familial cold autoinflammatory syndrome (FCAS) and 1 chronic infantile neurological cutaneous articular syndrome (CINCA)], 2 systemic juvenile idiopathic arthritis (sJIA), 1 idiopathic recurrent pericarditis and 1 pyoderma gangrenosum case. Mean duration of followup was $5.1 \pm 3.7$ years. Canakinumab was used for $21.8 \pm 15.8$ months (6-54 months). Overall beneficial clinical response to canakinumab, based on the expert opinion, was seen in 
93.1\% of the patients and canakinumab was discontinued only in 3 patients. These were one crFMF patient due to control of the symptoms, one sJIA and one pyoderma gangrenosum case due to persistence of the clinical features.

In crFMF patients, the most common reason for use of biologics was resistance to colchicine. In 7 of the patients, in addition to colchicine resistance, presence of subclinical inflammation was another indication for biologic use and one patient had renal amyloidosis. Table I shows demographic and clinical features of the crFMF patients. The crFMF patients had a female predominance; 16 girls and 3 boys. Mean age at the first symptoms of FMF was 2.8 \pm 2.2 years ( 3 months -7 years) and mean age at diagnosis of FMF and use of colchicine was 5.3 \pm 3.1 years (3 months - 11 years). Fifteen patients had M694V homozygous mutation, one patient had M694V/R761H compound heterozygous mutation and in 3 of the cases we could not have demonstrated a pathogenic mutation in the $M E F V$ and other monogenic periodic fever syndrome genes. In 14 of 19 crFMF patients, anakinra was used before canakinumab for $6.0 \pm 2.9$ months ( 3 - 12 months). The duration between the start of colchicine and interleukin-1 blocker was $4.3 \pm 2.6$ years ( $1-9$ years). Mean number of attacks per year before colchicine was $18.7 \pm 6.9(10-36)$, after colchicine it was $8.2 \pm 2.7(6-12)$ and after biologics the number dropped to $0.1 \pm 0.3(0-1)$ attacks per year. The most common reason for switching anakinra to canakinumab was painful daily injections. The mean dose of canakinumab was $4.0 \pm 0.9 \mathrm{mg} /$ $\mathrm{kg}(3-6 \mathrm{mg} / \mathrm{kg})$. At the time of enrollment, in 11 patients canakinumab was being used every month and in 8 patients every 3-4 months.

Clinical and demographic features of patients with HIDS-MKD and CAPS are shown in Table II. Canakinumab was used in three HIDS-MKD cases. Two of them were brothers and both had homozygous V377I mutation in the MVK gene, and the other patient had heterozygous V377I mutation. Initially anakinra was used for 7 months and canakinumab was being used for 10 months with resolution of the attacks. Two
FCAS patients were using canakinumab for 13 months with total disappearance of rash and fever attacks. CINCA patient did not show dramatic response to standard doses of both IL-1 blockers (anakinra and canakinumab) with partial control of the acute phase reactants (APRs). The dose of canakinumab was increased to $12 \mathrm{mg} / \mathrm{kg} / \mathrm{month}$ that made the control of APRs and clinical features. In one of the sJIA patients canakinumab was used for 27 months with good response. But other sJIA case initially followed a polycyclic course and for 28 months canakinumab was used with a good response but later turned into polyarticular course under canakinumab treatment. A severe pyoderma gangrenosum patient that failed dapson and anakinra also failed canakinumab that was used for 9 months. We have successfully treated a case of idiopathic recurrent pericarditis with canakinumab. No major adverse event was observed under canakinumab treatment. One patient had impetigo and five patients had upper respiratory tract infections. All canakinumab injections were well tolerated with no injection site reactions.

\section{Discussion}

The world of autoinflammation has seen breakthrough developments in the recent years with the description of new monogenic diseases. Better understanding of the pathophysiology of the diseases led to the discovery of more targeted, more selected therapies, namely cytokine blockers.,13

Familial Mediterranean fever is the prototype of autoinflammatory diseases and caused by gain of function mutations in the MEFV gene. Mutated pyrin protein leads to over activity of inflammasomes and production of abnormal amounts of inflammatory cytokine IL- $1 \beta ., 1,2,7$ Colchicine is the standard treatment option in FMF and prevents both the attacks and decreases the risk of amyloidosis. Less than 5\% of FMF patients do not show any improvement with colchicine. These patients are called as crFMF but there is no internationally accepted 


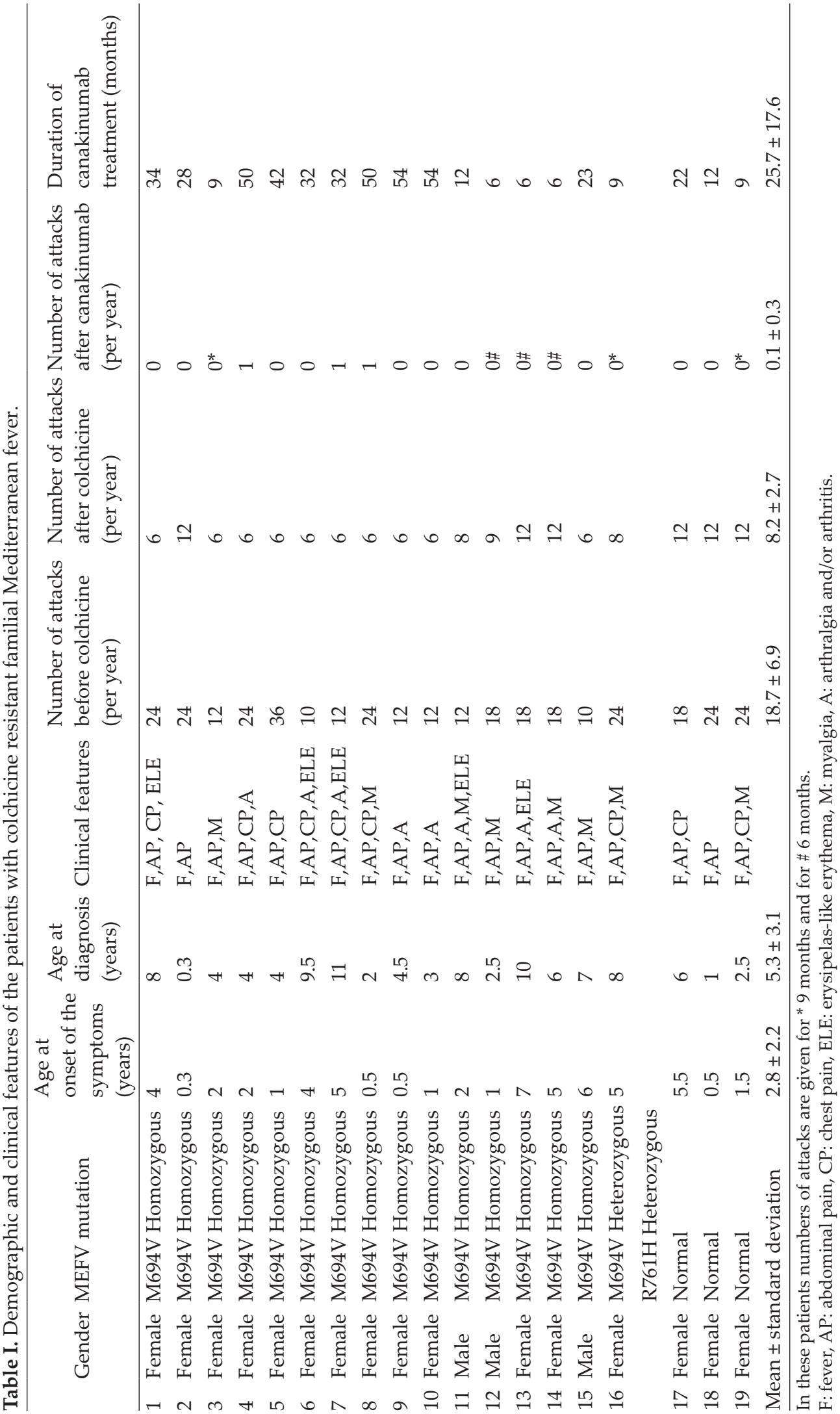


Table II. Demographic and clinical features of the patients with HIDS-MKD and CAPS.

\begin{tabular}{|c|c|c|c|c|c|c|}
\hline Diagnosis & Gene analysis & Gender & $\begin{array}{l}\text { Age at } \\
\text { the onset }\end{array}$ & $\begin{array}{l}\text { Age at the } \\
\text { diagnosis }\end{array}$ & Clinical features & $\begin{array}{l}\text { Duration of } \\
\text { canakinumab } \\
\text { treatment }\end{array}$ \\
\hline HIDS-MKD & $\begin{array}{l}\text { V377I } \\
\text { Homozygous }\end{array}$ & Male & 1 year & 5.5 years & $\begin{array}{l}\text { Fever, abdominal pain, diarrhea, } \\
\text { lymphadenopathy, oral ulcers, } \\
\text { splenomegaly }\end{array}$ & 9 months \\
\hline HIDS-MKD & $\begin{array}{l}\text { V377I } \\
\text { Homozygous }\end{array}$ & Male & 2 years & 9 years & $\begin{array}{l}\text { Fever, abdominal pain, arthralgia, } \\
\text { oral ulcers, painful lymphadenitis }\end{array}$ & 9 months \\
\hline HIDS-MKD & $\begin{array}{l}\text { V377I } \\
\text { Heterozygous }\end{array}$ & Male & 1 year & 2 years & $\begin{array}{l}\text { Fever, abdominal pain, rash, } \\
\text { diarrhea, vomiting, aphthous } \\
\text { stomatitis }\end{array}$ & 12 months \\
\hline CAPS (CINCA) & $\begin{array}{l}\text { V198M } \\
\text { Heterozygous }\end{array}$ & Female & Neonatal & 1.5 years & $\begin{array}{l}\text { Premature delivery, fever, rash, } \\
\text { arthropathy, developmental delay }\end{array}$ & 17 months \\
\hline CAPS (FCAS) & $\begin{array}{l}\text { Q703K } \\
\text { Heterozygous }\end{array}$ & Male & 7 years & 8 years & Fever, rash, myalgia, conjunctivitis & 15 months \\
\hline CAPS (FCAS) & $\begin{array}{l}\text { V198M } \\
\text { Heterozygous }\end{array}$ & Female & 8 years & 9 years & $\begin{array}{l}\text { Fever, rash, arthralgia, myalgia, } \\
\text { conjunctivitis }\end{array}$ & 11 months \\
\hline
\end{tabular}

HIDS-MKD: hyperimmunoglobulin D syndrome-mevalonate kinase deficiency, CAPS: cryopyrin-associated periodic syndrome, CINCA: chronic infantile neurological cutaneous articular syndrome, FCAS: familial cold autoinflammatory syndrome.

consensus definition for colchicine resistance. ${ }^{6}$ The most widely used one was defined by Hentgen et al. ${ }^{14}$ as having more than 6 typical FMF attacks per year or more than 3 typical attacks over a 4-6 month period. In cases where attacks are incomplete, then increases in at least 2 of 3 acute phase reactants (C-reactive protein, erythrocyte sedimentation rate, serum amyloid A) between attacks would identify the patient as colchicine resistant. The most important issue before calling an FMF patient as colchicine resistant is the compliance of the patient. ${ }^{6,8}$ Compliance was reassured in our institution by daily pill counting that was made by the parents. We did not have any colchicine intolerant patient in the cohort that may be a problem in $2-5 \%$ of FMF patients. ${ }^{8}$

It is known that FMF patients with exon 10 mutations have the most severe phenotype, especially the ones having M694V homozygous mutations. These patients tend to have initial symptoms of FMF at young ages, have more frequent attacks and need higher doses of colchicine. Nearly $25-30 \%$ of FMF patients have only one mutation in the MEFV gene and a small number of FMF patients do not exhibit any known pathogenic mutation in the $M E F V$ gene. ${ }^{1,5,8}$ In this study, 15 patients (78.9\%) had M694V homozygous mutation and the mean age at the first symptoms of FMF was 2.8 years, supporting the notion that FMF patients with homozygous exon 10 mutations have a severe course. One patient had compound heterozygous mutations in exon 10 (M694V/ $\mathrm{R} 761 \mathrm{H}$ ) and in 3 cases we were unable to demonstrate any pathogenic mutation in the $M E F V$ and other monogenic periodic fever syndrome genes, namely MVK, TNFRSF1A, and NLRP3. We have classified them under crFMF group, because these patients fulfilled criteria sets for FMF (both Eurofever and Turkish pediatric FMF criteria) and had classical symptoms of FMF without any peculiar signs and symptoms suggestive of another AID and had not a complete, but partial response to colchicine and had a dramatic response to IL-1 blockers. ${ }^{11,12}$

Elucidation of the role of IL-1 in the pathogenesis of FMF and other autoinflammatory diseases led to the discovery of IL-1 blockers. Anakinra $\left(\right.$ Kineret $\left.^{\circledR}\right)$ is a short-acting recombinant IL-1 receptor antagonist and blocks IL-1 $\alpha$ and IL-1 $\beta$ 
binding to the IL-1 receptor, and administered as daily subcutaneous injections. ${ }^{10}$ There are many case reports and case series on beneficial effects of anakinra in crFMF patients. ${ }^{15}$ Ben-Zvi et al. ${ }^{16}$ conducted a double-blind randomized placebo-controlled trial in 25 colchicine resistant adult FMF patients over 4 months of period. The attack rate, the primary outcome, was significantly less among the anakinra group versus placebo $(1.7 \pm 1.7$ versus $3.5 \pm$ 1,9 attacks per patient month, respectively, $p=0.037$ ). Başaran et al.$^{17}$ presented 8 cases of colchicine resistant pediatric FMF patients that all showed complete remission under anakinra treatment. The most problematic issue with anakinra is daily painful injections which is more problematic in children. So, in many of the manuscripts written on FMF and IL-1 blockers, anakinra was switched to canakinumab. ${ }^{17,18} \mathrm{We}$ have used anakinra before canakinumab in 14 of 19 crFMF patients with a mean duration of 6 months and have seen that anakinra was effective in all patients in both controlling the frequency of attacks and subclinical inflammation. Painful daily injections were the reason in all cases for switching to canakinumab.

Canakinumab (llaris $^{\circledR}$ ) is a recombinant fully humanized selective anti-IL-1 $\beta$ monoclonal antibody and binds to serum IL$1 \beta$ and neutralizes its activity by blocking the interaction with IL-1 receptors. It is administered subcutaneously every one or two months depending on the indication and has approval for CAPS and sJIA but not for crFMF in Europe and North America. ${ }^{1,9,10}$ There are two open-label phase II studies looking for efficacy and safety of canakinumab in crFMF patients. Both studies have shown that canakinumab was effective in reducing the frequency of FMF attacks and maintaining low levels of APRs with no unexpected adverse events.,19 Kara Eroglu et al. ${ }^{18}$ presented 9 cases of pediatric crFMF patients and all responded well to canakinumab. Başaran et al. ${ }^{17}$ presented 4 children with crFMF in whom canakinumab was used. In all of them anakinra was used initially. Three cases showed sustained remission under canakinumab but in one case canakinumab was switched back to anakinra after 3 months due to clinical and laboratory worsening. Recently published international CLUSTER study (The phase 3 canakinumab pivotal umbrella study in three hereditary periodic fevers) evaluated canakinumab efficacy in crFMF, HIDS-MKD and TRAPS patients and it was shown that at week $16,61 \%$ of patients with crFMF had complete response to canakinumab while this number was $6 \%$ on placebo group. ${ }^{3}$ We have used canakinumab in $19 \mathrm{crFMF}$ patients with a mean duration of 25.7 months, and in 6 of them canakinumab was used for less than a year. We have seen that canakinumab was effective both in short and long term in all cases in both controlling the attacks and APRs. There is no consensus definition for optimal dose interval and duration of canakinumab treatment in crFMF patients. ${ }^{9}$ We have used canakinumab every month for 6 months at the beginning of the treatment and then every 2-4 months depending on the severity of the previous FMF attacks and have observed that decreasing the intervals of injections did not lead to reemergence of the FMF attacks in the majority of the patients. Only 3 patients had mild FMF attacks once per year without necessitating increasing the frequency of injections.

The other two autoinflammatory diseases that canakinumab was used in this cohort were HIDS-MKD and CAPS. Life-long IL-1 inhibition is indicated in patients with CAPS. In HIDS-MKD patients with frequent attacks and/ or subclinical inflammation between attacks, maintenance therapy with IL-1 blockade or etanercept is recommended. ${ }^{20,21}$ Two FCAS cases showed dramatic response to standard doses of canakinumab (2 mg/kg/2 months) with total resolution of rash and constitutional symptoms. But CINCA patient did not show the same response to both anakinra and canakinumab. The symptoms and APRs were controlled only with very high dose of canakinumab $(12 \mathrm{mg} / \mathrm{kg} /$ month). Kuemmerle-Deschner et al. ${ }^{22}$ reported that real-life effectiveness of canakinumab in daily clinical practice of CAPS was lower $(53 \%$ 
with standart dose and $72 \%$ after dose increase) than reported in a placebo-controlled, doubleblinded trial, in which complete remission was achieved in $97 \%$ of patients. Russo et al. ${ }^{23}$ used canakinumab in 10 CAPS (8 MuckleWells syndrome and 2 CINCA) patients. Canakinumab was used for a median of 21 months and clinical remission was obtained in all patients. Sixty percent of the patients needed dose adjustments and CINCA cases required higher doses of canakinumab than those with Muckle-Wells syndrome. HIDS-MKD patients are treated depending on the frequency of attacks. TNF-alpha inhibitors and IL-1 blockers (both anakinra and canakinumab) have been used with variable success. Case reports and case series have shown that $80 \%$ of HIDS-MKD patients improved using anakinra continuously or during attacks only. ${ }^{24}$ The study of Galeotti et al. ${ }^{25}$ demonstrated that in 11 patients with HIDS-MKD, continuous IL-1 blocking was associated with complete remission in 4 cases and partial remission in seven. They have stated that IL-1 blockade brings substantial benefit to HIDS-MKD patients. We initially used anakinra and later canakinumab in 3 HIDS-MKD cases and all showed dramatic resolution of attacks and normalization of APRs under IL-1 blocking regimens, confirming a key role of IL-1 in HIDSMKD pathogenesis.

Systemic JIA is characterized by fever, rash, arthritis, serositis, lymphadenopathy and hepatosplenomegaly. Early use of biologics (anakinra, canakinumab or tocilizumab) is recommended in patients with severe disease course or impending macrophage activation syndrome. ${ }^{26}$ The study of Ruperto et al. ${ }^{27}$ reported that $76 \%$ of 190 sJIA patients attained ACR Pedi-90 response with canakinumab at the end of the withdrawal phase. We have used canakinumab in 2 sJIA patients. Both cases had a severe course complicated with macrophage activation syndrome. One case followed a polycyclic course and canakinumab resulted in resolution of sJIA flares. But the other case initially followed a polycyclic course and later turned into polyarticular course under canakinumab treatment.
This is one of the manuscripts that include high number of crFMF patients on canakinumab with relatively long duration of follow-up time. Canakinumab was used in 29 children and only in 2 cases discontinued due to inefficacy. Overall efficacy of canakinumab was $93.1 \%$ in this study. In conclusion, canakinumab seems to be effective, safe and well tolerated in children with rheumatic diseases. In patients with crFMF, monthly canakinumab injections may be decreased to every other month after the completion of initial six months.

\section{REFERENCES}

1. Barron KS, Kastner DL. Periodic fever syndromes and other inherited autoinflammatory diseases. In: Petty RE, Laxer RM, Lindsey CB, Wedderburn LR (eds). Textbook of Pediatric Rheumatology (7th ed) Philadelphia: Elsevier, 2016: 609-626.

2. Manthiram K, Zhou Q, Aksentijevich I, Kastner DL. The monogenic autoinflammatory diseases define new pathways in human innate immunity and inflammation. Nat Immunol 2017; 18: 832-842.

3. De Benedetti F, Gattorno M, Anton J, et al. Canakinumab for the treatment of autoinflammatory recurrent fever syndromes. N Engl J Med 2018; 378: 1908-1919.

4. Oda H, Kastner DL. Genomics, biology, and human illness: advances in the monogenic autoinflammatory diseases. Rheum Dis Clin North Am 2017; 43: 327345 .

5. Omenetti A, Carta S, Delfino L, Martini A, Gattorno M, Rubartelli A. Increased NLRP3-dependent interleukin $1 \beta$ secretion in patients with familial Mediterranean fever: correlation with MEFV genotype. Ann Rheum Dis 2014; 73: 462-469.

6. Ozen S, Kone-Paut I, Gül A. Colchicine resistance and intolerance in familial Mediterranean fever: definition, causes, and alternative treatments. Semin Arthritis Rheum 2017; 47: 115-120.

7. Gül A, Ozdogan H, Erer B, et al. Efficacy and safety of canakinumab in adolescents and adults with colchicine-resistant familial Mediterranean fever. Arthritis Res Ther 2015; 17: 243.

8. Özen S, Batu ED, Demir S. Familial Mediterranean fever: recent developments in pathogenesis and new recommendations for management. Front Immunol 2017; 8: 253.

9. Haviv R, Hashkes PJ. Canakinumab investigated for treating familial Mediterranean fever. Expert Opin Biol Ther 2016; 16: 1425-1434. 
10. Grattagliano I, Bonfrate L, Ruggiero V, Scaccianoce G, Palasciano G, Portincasa P. Novel therapeutics for the treatment of familial Mediterranean fever: from colchicine to biologics. Clin Pharmacol Ther 2014; 95: 89-97.

11. Yalçınkaya F, Özen S, Özçakar ZB, et al. A new set criteria for diagnosis of familial Mediterranean fever in childhood. Rheumatology (Oxford) 2009; 48: 395398.

12. Federici S, Sormani MP, Ozen S, et al; Paediatric Rheumatology International Trials Organisation (PRINTO) and Eurofever Project Evidence-based provisional clinical classification criteria for autoinflammatory periodic fevers. Ann Rheum Dis 2015; 74: 799-805

13. Jesus AA, Goldbach-Mansky R. IL-1 blockade in autoinflammatory syndromes. Annu Rev Med 2014; 65: 223-244.

14. Hentgen V, Grateau G, Kone-Paut I, et al. Evidence-based recommendations for the practical management of familial Mediterranean fever. Semin Arthritis Rheum 2013; 43: 387-391.

15. Van der Hilst JCH, Moutschen M, Messiaen PE, Lauwerys BR, Vanderschueren S. Efficacy of antiIL-1 treatment in familial Mediterranean fever: a systematic review of the literature. Biologics 2016; 10: 75-80.

16. Ben-Zvi I, Kukuy O, Giat E, et al. Anakinra for colchicine-resistant familial Mediterranean fever: a randomized, double-blind, placebo-controlled trial. Arthritis Rheumatol 2017; 69: 854-862.

17. Başaran Ö, Uncu N, Çelikel BA, Taktak A, Gür $\mathrm{G}$, Çakar N. Interleukin-1 targeting treatment in familial Mediterranean fever: an experience of pediatric patients. Mod Rheumatol 2015; 25: 621-624.

18. Kara Eroğlu F, Beşbaş N, Topaloğlu R, Ozen S. Treatment of colchicine-resistant Familial Mediterranean fever in children and adolescents. Rheumatol Int 2015; 35: 1733-1737.
19. Brik R, Butbul-Aviel Y, Lubin S, et al. Canakinumab for the treatment of children with colchicine-resistant familial Mediterranean fever: a 6-month open-label, single-arm pilot study. Arthritis Rheumatol 2014; 66: 3241-3243.

20. ter Haar NM, Oswald M, Jeyaratnam J, et al. Recommendations for the management of autoinflammatory diseases. Ann Rheum Dis 2015; 74: 1636-1644.

21. Çakan M, Aktay-Ayaz N, Keskindemirci G, Karadağ ŞG. Two cases of periodic fever syndrome with coexistent mevalonate kinase and Mediterranean fever gene mutations. Turk J Pediatr 2017; 59: 467470.

22. Kuemmerle-Deschner JB, Hofer F, Endres T, et al. Real-life effectiveness of canakinumab in cryoprinassociated periodic syndrome. Rheumatology (Oxford) 2016; 55: 689-696.

23. Russo RAG, Melo-Gomes S, Lachmann HL, et al. Efficacy and safety of canakinumab therapy in paediatric patients with cryopyrin-associated periodic syndrome: a single-centre, real-world experience. Rheumatology (Oxford) 2014; 53: 665670.

24. Moll M, Kuemmerle-Deschner JB. Inflammasome and cytokine blocking strategies in autoinflammatory disorders. Clin Immunol 2013; 147: 242-275

25. Galeotti C, Meinzer U, Quartier P, et al. Efficacy of interleukin-1-targeting drugs in mevalonate kinase deficiency. Rheumatology (Oxford) 2012; 51: 18551859.

26. Benedetti FD, Schneider R. Systemic juvenile idiopathic arthritis. In: Petty RE, Laxer RM, Lindsey CB, Wedderburn LR (eds). Textbook of Pediatric Rheumatology (7th ed) Philadelphia: Elsevier, 2016: 205-216.

27. Ruperto N, Brunner HI, Quartier P, et al; for the PRINTO and PRCSG. Two randomized trials of canakinumab in systemic juvenile idiopathic arthritis. N Eng J Med 2012; 367: 2396-2406. 